\title{
In situ absolute calibration of a channel electron multiplier for detection of positive ions
}

\author{
D. W. Savin, ${ }^{\text {a) }}$ L. D. Gardner, D. B. Reisenfeld, A. R. Young, ${ }^{\text {b) }}$ and J. L. Kohl \\ Harvard-Sinithsonian Center for Astrophysics, Cambridge, Massachusetts 02138
}

(Received 21 July 1994; accepted for publication 7 October 1994)

\begin{abstract}
A technique has been developed to determine the absolute single particle counting efficiency of a channel electron multiplier (CEM) for positively charged ions at $\mathrm{keV}$ energies. The calibration technique is applicable to positive ion detectors in general. Ion beam currents of $\mathrm{C}^{2+}$ were created by charge transfer of $\mathrm{C}^{3+}$ on $\mathrm{H}_{2}$ in the thin target regime. By adjusting the $\mathrm{H}_{2}$ pressure in the beam scattering chamber from $10^{-10}$ to $10^{-6}$ Torr, $\mathrm{C}^{2+}$ currents could be created either low enough to be detected by the CEM in the particle counting mode or high enough to be measured as a current using the CEM as a Faraday cup. The CEM counting efficiency was determined by comparing the $\mathrm{C}^{2+}$ count rate to the $\mathrm{C}^{2+}$ current, scaling by the change in $\mathrm{H}_{2}$ pressure, and also scaling by the incident $\mathrm{C}^{3+}$ current for each $\mathrm{C}^{2+}$ measurement. This method, which effectively uses the charge transfer cross section as a "transfer standard," allows the CEM counting efficiency to be determined in situ and to be monitored accurately over extended periods of time. The calibration requires that only the relative, not the absolute, pressure change be known. The technique does not require the value of the charge transfer cross section to be known. The accuracy of the calibration technique for the present tesults was $10 \%$ at a confidence level considered to be equivalent to a statistical $90 \%$ confidence level. For the present work, the peak counting efficiency of a Galileo $4039 \mathrm{CEM}$ for $32.5 \mathrm{keV} \mathrm{C}^{2+}$ ions was determined to be $96 \%$. (C) 1995 American Institute of Physics.
\end{abstract}

\section{INTRODUCTION}

The channel electron multiplier (CEM) is a commonly used detector for particles and photons. The high gain, low noise, simplicity, and compact size of CEMs make them suitable for a wide range of applications. A general overview of the characteristics and applications of CEMs has been given by Kurz. ${ }^{1}$ Absolute single particle detection (i.e., counting) efficiencies have been reported for electrons, ${ }^{2-7}$ ultraviolet (UV) photons, $\mathrm{x}$ rays, and $\gamma$ rays (reviewed by Macau et al. ${ }^{7}$, and ions. ${ }^{2-4,7-13}$

The present work was motivated by the need to determine the absolute detection efficiency of a Galileo 4039 CEM for $32.5 \mathrm{keV} \mathrm{C} \mathrm{C}^{2+}$ ions within the tight constraints of our experimental requirements. The need for ultrahigh vacuums $\left(10^{-10}-10^{11}\right.$ Torr) required baking the interaction chamber (and hence the CEM) for $72 \mathrm{~h}$ at $200^{\circ} \mathrm{C}$. Thus, a way was needed to calibrate the CEM in situ after it had been baked. Also, the high counting rates $\left(\geq 1 \times 10^{5} \mathrm{~s}^{-1}\right)$ associated with the experiment required a calibration method that allowed the long term behavior of the CEM to be monitored accurately.

Two basic calibration techniques have been described in the literature. The scatter in reported peak detection efficiencies illustrates the difficulty and importance of making an accurate efficiency determination. The more common calibration technique ${ }^{2-4,8,11,12,14,15}$ is to place the CEM behind a Faraday cup which has a small hole in it. The CEM efficiency can then be determined by measuring the CEM count

\footnotetext{
a) Present address: Department of Physics, University of California, Berkeley, CA 94720.

b) Present address: Physics Department-Jadwin Hall, Princeton University, Princeton, NJ 08544.
}

rate, the hole size, the density profile of the ion beam, and the total beam current. The less common technique $e^{9,10,13}$ is to use ion beam currents low enough to be counted in the CEM $\left(10^{-14}-10^{-15} \mathrm{~A}\right)$ and then to be measured as a current using either the CEM as a Faraday cup or using a separate Faraday cup. Both of these calibration techniques, though, can be difficult or inconvenient to apply in a complex experimental arrangement.

The optimal experimental situation is that the CEM be calibrated under conditions as close as possible to actual data collection conditions. In applications that involve ultrahigh vacuums and alignments critical for reasons of ion optics, this requires leaving the CEM in situ and under vacuum. In such cases the ion optics of the experiment may make it difficult to determine the density profile of the beam near the CEM and also difficult to implement the insertion and retraction of the needed Faraday cup, as required by the more common calibration technique. In the case of the second calibration technique, the required measurement of femptoampere currents can be difficult, as leakage currents and background induced currents often compete with the low ion beam currents to be measured. Because of the difficulty of implementing either of the calibration techniques reported in the literature and because of our need for an in situ calibration technique which is reliable for extended periods of time, we have developed a calibration technique which uses the chàrge transfer cross section of $\mathrm{C}^{3+}$ on $\mathrm{H}_{2}$ as a "transfer standard."

\section{EXPERIMENTAL ARRANGEMENT}

Although the calibration technique described here is generally applicable, it will be described in terms of an apparatus designed for measurements of electron impact exci- 


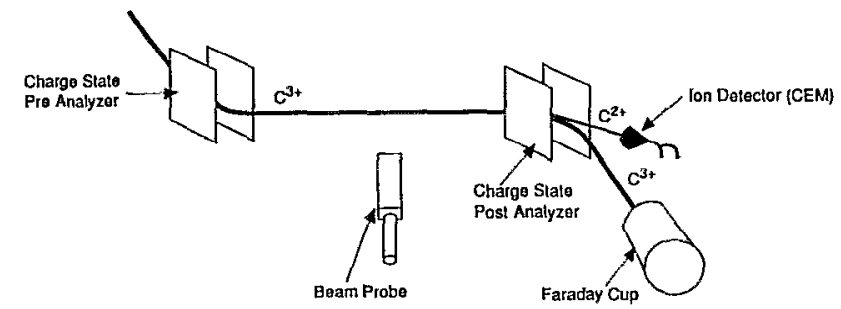

FIG. 1. Diagram of the experimental apparatus. The path length of the ion beam between the preanalyzer and post-analyzer is $21 \pm 3 \mathrm{~cm}$.

tation, charge transfer, and dielectronic recombination. ${ }^{16} \mathrm{~A}$ diagram of the experimental apparatus is shown in Fig. 1. $\mathrm{C}^{3+}$ is created in a Penning ion source, charge-to-mass selected, formed into a beam, and transported to the scattering chamber. In the scattering chamber the $\mathrm{C}^{3+}$ passes through an electrostatic charge state "preanalyzer" which removes any $\mathrm{C}^{2+}$ created by surface scattering or by charge transfer upstream of the preanalyzer and directs the purified $C^{3+}$ beam toward the scattering volume. A Faraday cup beam probe in the center of the collision chamber allows the shape and total current of the ion beam to be determined. The ions continue into an electrostatic charge state "post-analyzer" which separates the $\mathrm{C}^{3+}$ from any $\mathrm{C}^{2+}$ which was created downstream of the preanalyzer. The $\mathrm{C}^{3+}$ is detected using a Galileo 4039 CEM as a Faraday cup (Fig. 2) or a separate Faraday cup. The $\mathrm{C}^{2+}$ is detected either using the CEM in the particle counting mode (Fig. 3) or as a Faraday cup. Located $4 \mathrm{~cm}$ in front of the CEM is an electrically isolated aperture with a $1-\mathrm{cm}$-diam opening. Any beam current that strikes the aperture can be detected. Located $0.5 \mathrm{~cm}$ in front of the CEM is an electrically isolated grid with a $90 \%$ transmittance as determined geometrically. The mouth of the CEM is $1 \mathrm{~cm}$ in diameter.

To use the CEM.as a Faraday cup (Fig. 2), a Keithley 602 Electrometer was connected to the CEM entrance cone.

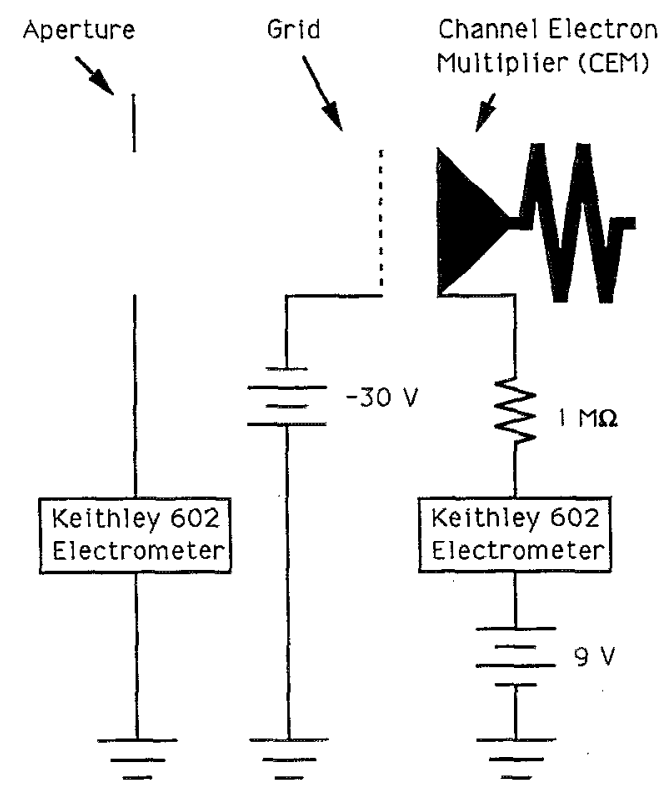

FIG. 2. The CEM used as a Faraday cup.

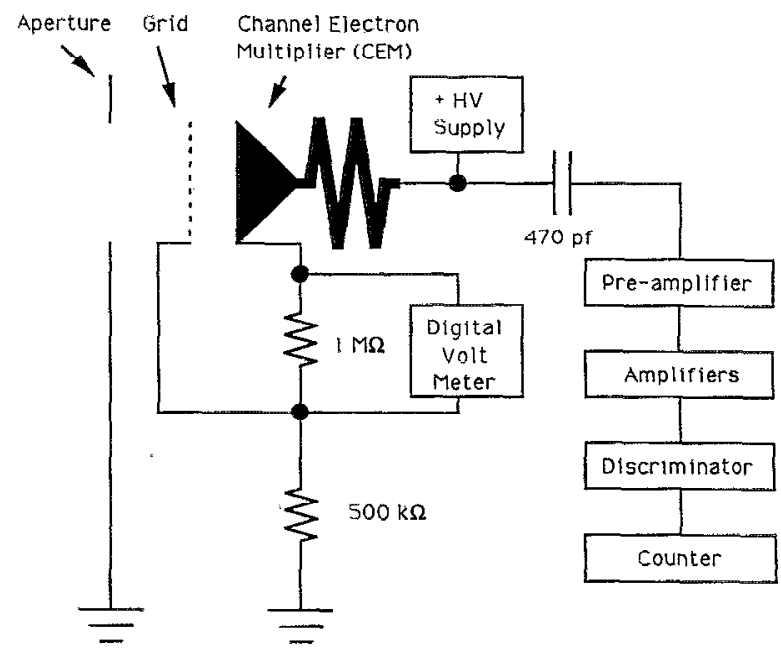

FIG. 3. The CEM as used for particle counting.

A potential of $-30 \mathrm{~V}$ was applied to the grid to minimize the loss of secondary electrons from the CEM cone. The CEM was also biased $+9 \mathrm{~V}$ to minimize further the loss of secondary electrons. No electrical connections were made to the CEM tail in order to prevent possible leakage currents or paths to ground other than through the electrometer. To determine that the CEM was collecting the entire ion beam, the beam was scanned horizontally and vertically across the CEM and the currents from the CEM and aperture were monitored.

The efficiency of the CEM as a Faraday cup was determined by comparing the current measured by the CEM (correcting for the grid in front of the CEM) to the total ion beam current as measured with the beam probe. The ion beam was sampled at points in space $0.2-0.5 \mathrm{~mm}$ apart using the probe Faraday cup which has an aperture $\approx 0.25 \mathrm{~mm}$ in diameter. The current flux at each point was computed by dividing the measured current by the area of the aperture. The Faraday cup of the beam probe was biased positively to minimize the loss of secondary electrons. The optimal bias voltage ( $+9 \mathrm{~V})$ was determined while measuring the beam probe Faraday cup current and increasing the bias voltage until no further change was detected in the measured current. The total beam current was determined by integrating the flux measurements. The accuracy of the comparison between the two current measurements was limited by the uncertainty in the beam probe aperture area ( $7 \%$ at the $90 \%$ confidence level), by the uncertainty in the beam probe biasing technique $(2 \%)$, and by the ion beam stability (3\%). Treating these as random-sign errors and adding them in quadrature yields an $8 \%$ total uncertainty. An efficiency of $100 \%$ for the CEM as a Faraday cup was consistent with the results and was adopted for the present calibration.

For single particle counting (Fig. 3), a positive bias voltage $(2.3-3.3 \mathrm{kV})$ was applied to the CEM tail and the signal pulses were capacitively coupled to an amplifier chain consisting of an Ortec 9301 preamplifier and two stages of an EG\&G AN201/N quad amplifier. The output of the final amplifier was connected to an Ortec 473 constant fraction discriminator which was in turn connected to an Ortec 772 
counter. The grid and cone voltages were kept nearly identical to help insure a uniform detection response across the CEM opening. ${ }^{17,18} \mathrm{~A} 1 \mathrm{M} \Omega$ resistor between the grid and the CEM caused an insignificant voltage difference $(\approx 4 \mathrm{~V})$ between the two. The dc bias current through the CEM was monitored as a function of counting rate by measuring the voltage drop across this $1 \mathrm{M} \Omega$ resistor which was connected in series between the CEM cone and ground. The counting response of a CEM is expected to become nonlinear for incident particle rates large enough to change the bias current by more than $10 \% .{ }^{19}$ Because of the high counting rates (up to $3 \times 10^{5} \mathrm{~s}^{-1}$ ) associated with the experiment, it was necessary to run the CEM in a "low-gain" mode to ensure linearity of the CEM counting response. The CEM was therefore operated at the edge of the plateau on the count-rate versus detector-voltage curve. Hence, a slight degradation in the gain could cause a significant change in the CEM detection efficiency. The ability to monitor the efficiency over time, thus, became crucial to the experiment. Most of the CEM calibration reported below was carried out at high count rates with the CEM run in the low-gain mode in order to simulate experimental operating conditions.

Previously reported CEM calibration techniques for ion detection (described in Sec. I) show that detection efficiencies near unity are expected for ion energies $\geq 3 \mathrm{keV}$. Those reported results were carried oul with CEMs run in a "highgain" mode. To allow the present CEM calibration and the technique to be compared with these previous results, the CEM was also calibrated at low count rates $\left(\Sigma 1 \times 10^{5}\right)$ in a high-gain mode. This allowed the peak CEM detection efficiency to be determined. The only real difference between the high-gain and low-gain modes was the bias voltage applied to the CEM. In the low-gain mode the CEM was run at voltages between 2.3 and $2.7 \mathrm{kV}$ while in the high-gain mode the CEM was run at voltages of up to $3.3 \mathrm{kV}$. A pulse height analysis was performed to select the CEM operating voltage, amplifier settings, and discriminator levels for each mode.

\section{CALIBRATION TECHNIQUE}

Charge transfer of $\mathrm{C}^{3+}$ on $\mathrm{H}_{2}$ can be written as

$$
\mathrm{C}^{3+}+\mathrm{H}_{2} \rightarrow \mathrm{C}^{2+}+\left[\mathrm{H}_{2} \text { products }\right]^{+} \text {. }
$$

To calibrate the CEM, it was first used in the particle counting mode to detect charge transfer created $\mathrm{C}^{2+}$ ions in the thin target regime. The rate of detected $\mathrm{C}^{2+}$ ions is given by

$$
R_{\mathrm{C}^{2+}}=\epsilon \sigma \ln _{\mathrm{H}_{2}}(p) \frac{I_{\mathrm{C}^{3+}}}{3 e},
$$

where $R_{C^{2+}}$ is the $\mathrm{C}^{2+}$ detection rate, $\epsilon$ is the detection efficiency of the CEM, $\sigma$ is the charge transfer cross section for $32.5 \mathrm{keV} \mathrm{C}^{3+}$ ions on $\mathrm{H}_{2}, l$ is the path length of the ion beam through the gas, $n_{\mathrm{H}_{2}}(p)$ is the number density of $\mathrm{H}_{2}$ molecules at an $\mathrm{H}_{2}$ pressure $p, I_{\mathrm{C}^{3+}}$ is the $\mathrm{C}^{3+}$ current, and $e$ is the unit charge. To account for any $\mathrm{C}^{2+}$ created by charge transfer with gas other than hydrogen in the interaction chamber or by surface scattering, $R_{\mathrm{C}^{2+}}$ was measured as a function of the change in $\mathrm{H}_{2}$ pressure. The change in the $\mathrm{C}^{2+}$ rate, $\Delta R_{\mathrm{C}^{2+}}$, is given by

$$
\Delta R_{\mathrm{C}^{2+}}=\epsilon \sigma \ln _{\mathrm{H}_{2}}(\Delta p) \frac{I_{\mathrm{C}^{3+}}}{3 e},
$$

where $\Delta p$ is the $\mathrm{H}_{2}$ pressure change.

The pressure was measured using a Varian UHV-24 Bayard-Alpert-type nude ionization gauge. The gauge is expected to have a linear response from $2 \times 10^{-10}$ to $1 \times 10^{-5}$ Torr. ${ }^{20,21}$ The ion gauge was calibrated for measuring $\mathrm{N}_{2}$ pressures. The actual change in $\mathrm{H}_{2}$ pressure, $\Delta p$, is given by

$$
\Delta p=\frac{\Delta p_{g}}{G},
$$

where $\Delta p_{g}$ is the pressure change measured by the ion gauge, and the gauge constant $G$ is the sensitivity of the gauge for $\mathrm{H}_{2}$ relative to its sensitivity for $\mathrm{N}_{2}{ }^{20}$

Ideally it should be possible from Eq. (3) to determine $\epsilon$. Given the charge transfer cross section, the path length, and the $\mathrm{H}_{2}$ number density, then one could determine $\epsilon$ by measuring the $\mathrm{C}^{2+}$ rate and the $\mathrm{C}^{3+}$ current. However, the charge transfer cross section is not known well enough to use that approach. Charge transfer of $\mathrm{C}^{3+}$ on $\mathrm{H}_{2}$ and $\mathrm{D}_{2}$, near the energy of interest here, has been studied by a number of experimenters ${ }^{22-25}$ and reported cross section values differ by nearly a factor of 2 .

To avoid any uncertainties due to the range of reported charge transfer cross sections, we carried out a series of charge transfer measurements at pressures in the $10^{-6}$ Torr range, using the CEM as a Faraday cup, and measuring the charge transfer created current $I_{\mathrm{C}^{2+}}^{\prime}$ as a function of the $\mathrm{H}_{2}$ pressure change $\Delta p^{\prime}$. Following the example of Eq. (3) we have

$$
\Delta I_{\mathrm{C}^{2+}}^{\prime}=\frac{2}{3} \sigma \ln _{\mathrm{H}_{2}}\left(\Delta p^{\prime}\right) I_{\mathrm{C}^{3+}}^{\prime},
$$

where the CEM efficiency as a Faraday cup has been taken to be $100 \%$ (see Sec. II). Using the ideal gas law we can determine the increase in the $\mathrm{H}_{2}$ number density versus pressure change

$$
n_{\mathrm{H}_{2}}(\Delta p)=\frac{\Delta p}{k T},
$$

where $k$ is the Boltzmann constant and $T$ the temperature. Equations (3)-(6) can then be combined to yield the absolute CEM efficiency

$$
\epsilon=2 e \frac{I_{\mathrm{C}^{3+}}^{\prime}}{I_{\mathrm{C}^{3+}}}\left(\frac{\Delta R_{\mathrm{C}^{2+}}}{\Delta p_{g}} / \frac{\Delta I_{\mathrm{C}^{2+}}}{\Delta p_{g}^{\prime}}\right) .
$$

There are several advantages to this method for determining the CEM counting efficiency. First, the quantities on the right-hand side of Eq. (7) are all easily measurable. Second, the effects of the grid in front of the CEM, present for the $\Delta R_{\mathrm{C}^{2+}}, I_{\mathrm{C}^{3+}}, \Delta I_{\mathrm{C}^{2+}}^{\prime}$, and $I_{\mathrm{C}^{3+}}^{\prime}$ measurements, cancel out. Third, and most importantly, the parameters least accurately known (the charge transfer cross section $\sigma$, the path length $l$, and the absolute $\mathrm{H}_{2}$ pressure) cancel out in Eq. (7). Lastly, $\Delta R_{\mathrm{C}^{2}}$ and $I_{\mathrm{C}^{3}}$ do not need to be measured close in time to when $\Delta I_{\mathrm{C}^{2+}}^{\prime}$ and $I_{\mathrm{C}^{3+}}^{\prime}$ are measured. This allows for the counting efficiency to be monitored over extended periods of time. 


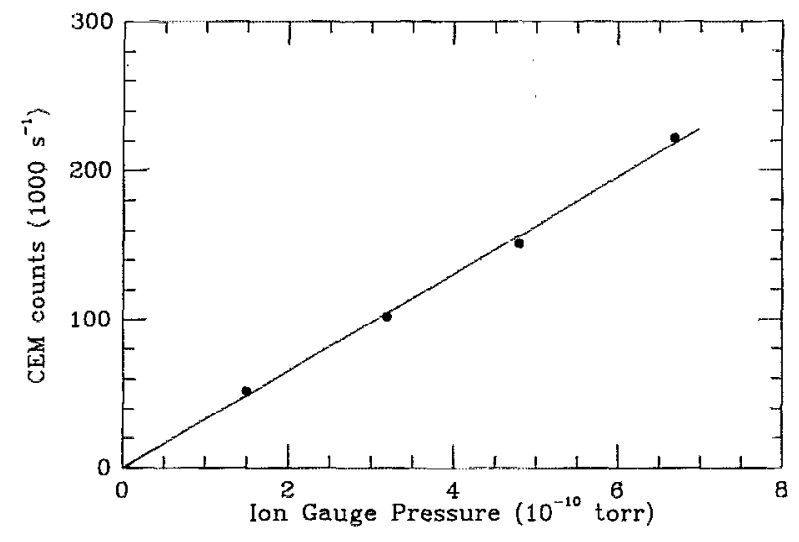

FIG. 4. CEM counts vs ion gauge pressure. The pressure reading has not been adjusted by the gauge constant for $\mathrm{H}_{2}$. The slope of the data, $\Delta R_{\mathrm{C}^{2+}} / \Delta p_{g}$, is $32.6 \times 10^{3}$ counts per second per $10^{-10}$ Torr for a $0.161 \mu \mathrm{A}$ beam of $C^{j+}$. A linear fit to the data is shown.

In fact, the only requirements for the validity of Eq. (7) are that the entire $\mathrm{C}^{2+}$ and $\mathrm{C}^{3+}$ beams be collected by the CEM and that the ionization gauge response be linear over the pressure range of interest and remain constant over time.

\section{RESULTS}

The CEM was calibrated in the low-gain and high-gain modes described in Sec. II. A typical plot of the number of $\mathrm{C}^{2+}$ ions detected by the CEM in the low-gain mode is shown in Fig. 4 as a function of ion gauge pressure (in the $10^{-10}$ Torr range). Data were taken (not shown) which demonstrated that the CEM response in the low-gain mode was linear up to count rates of nearly $3 \times 10^{5} \mathrm{~s}^{-1}$. Figure 5 shows a typical plot of the $\mathrm{C}^{2+}$ current measured by the CEM as a function of ion gauge pressure in the $10^{-6}$ Torr range. The data are corrected for a small $\left(\approx 0.04 \mathrm{nA}\right.$ per $10^{-6}$ Torr $)$ systematic positive background current. This background current had a linear dependence on pressure and was associated with the ion pump on the scattering chamber.

The CEM detection efficiency was determined from a number of data runs similar to those shown in Figs. 4 and 5.

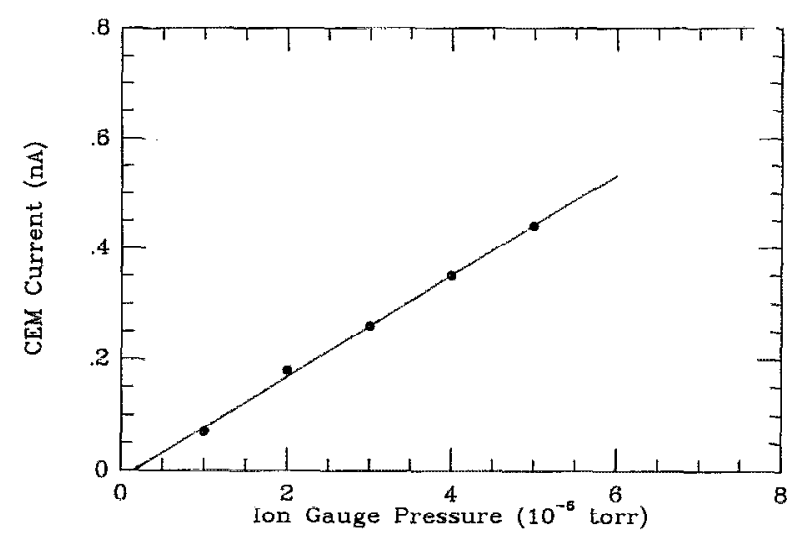

FIG. 5. CEM current vs ion gauge pressure. The pressure reading has not been adjusted by the gauge constant for $\mathrm{H}_{2}$. The slope of the data, $\Delta I_{\mathrm{C}^{2}+}^{\prime} / \Delta p_{g}^{\prime}$, is $0.091 \mathrm{nA}$ per $10^{-6}$ Torr for a $0.112 \mu \mathrm{A}$ beam of $\mathrm{C}^{3+}$. A linear fit to the data is shown.
TABLE I. Summary of uncertainties. All uncertainties are quoted at a confidence level which is taken to be equivalent to a statistical $90 \%$ confidence level.

\begin{tabular}{ll}
\hline \hline Aperture area of the beam probe & $7 \%$ \\
Beam probe biasing & $2 \%$ \\
Pressure stability & $6 \%$ \\
Ion beam stability & $3 \%$ \\
& \\
Quadrature sum & $10 \%$ \\
\hline \hline
\end{tabular}

In the high-gain mode, the peak CEM efficiency was determined to be $96 \%$. The high counting rates encountered during the experiment required that the CEM be run at low gain to prevent its counting rate response from becoming nonlinear. For the present work, in the low-gain mode, an efficiency of $80 \%$ was initially determined. During the time over which data were collected, the efficiency dropped to $56 \%$, but a sizable fraction of the loss in detection efficiency could be recovered by increasing the bias voltage applied to the CEM. This behavior is typical of a CEM as it ages.

The uncertainties associated with the CEM calibration are listed in Table I. Uncertainties are quoted at a confidence level which is taken to be equivalent to a statistical $90 \%$ confidence level. The accuracy of the beam probe measurements of the total $\mathrm{C}^{3+}$ current is discussed in Sec. II. It is estimated ${ }^{26}$ that $3 \%$ of the measured beam current is actually $\mathrm{O}^{4+}$. This contamination is not a source of uncertainty for the CEM calibration because it is expected to affect the initial currents $I_{\mathrm{C}^{3+}}$ and $I_{\mathrm{C}^{3+}}^{\prime}$ equally and so the contamination factor cancels out in Eq. (7). Similarly, the effect of any excited states of $\mathrm{C}^{3+}$ in the ion beam would also cancel out in Eq. (7). $\mathrm{O}^{4+}$ can also charge transfer off the added $\mathrm{H}_{2}$ gas creating $\mathrm{O}^{3+}$. However, $\mathrm{O}^{3+}$ is not expected to contaminate the $\mathrm{C}^{2+}$ signal because the resolving power of the postanalyzer is sufficient to separate the $\mathrm{C}^{2+}$ from the $\mathrm{O}^{3+}$. The dominant uncertainty in the pressure measurements was the accuracy to which the $\mathrm{H}_{2}$ pressure could be kept stable. Treating the uncertainties as random-sign errors and adding them in quadrature yields a total uncertainty of $10 \%$. This uncertainty of $10 \%$ does not represent the maximum accuracy of the present calibration technique. With some small improvements to the beam probe and the pressure stability, the accuracy of the calibration technique could easily be improved by at least a factor of 2 .

As a check on the calibration technique, it was possible to use Eq. (5) to determine the $\mathrm{C}^{3+}$ on $\mathrm{H}_{2}$ charge transfer cross section. The value of the gauge constant used $(0.48$ $\pm 0.06)$ was the average from a range of quoted gauge constants for $\mathrm{H}_{2}{ }^{20}$ The major uncertainties in determining the charge transfer cross section are due to the path length (14\%), the $\mathrm{H}_{2}$ pressure reading (6\%), the total beam current $(7 \%)$, the $\mathrm{O}^{4+}$ contamination factor $(1 \%)$, and the gauge constant (estimated to be $15 \%$, although it may be larger). Adding these uncertainties in quadrature results in a $23 \%$ total uncertainty. The charge transfer cross section determined from Eq. (5) was $(8.9 \pm 2.0) \times 10^{-16} \mathrm{~cm}^{2}$. This value lies within the range of reported cross sections. ${ }^{22-25}$ 


\section{ACKNOWLEDGMENTS}

This work was supported by NASA Supporting Research and Technology Program in Solar Physics Grant No. NAGW-1687.

${ }^{1}$ E. A. Kurz, Am. Lab. 11, 67 (1979).

${ }^{2}$ J. R. Sharber, J. D. Winnigham, and W. R. Sheldon, IEEE Trans. Nucl. Sci. NS-15, 536 (1968).

${ }^{3}$ A. Egidi, R. Marconero, G. Pizella, and F. Sperli, Rev. Sci. Instrum. 40, 88 (1969).

${ }^{4}$ B. Tatry, J. M. Bosqued, and H. Reme, Nucl. Instrum. Methods 69, 254 (1969).

${ }^{5}$ G. Paschmann, E. G. Shelley, C. R. Chappell, R. D. Sharp, and L. F. Smith, Rev. Sci. Instrum. 41, 1706 (1970).

${ }^{\circ}$ A. L. Bennani, J. Pebay, and B. Nguyen, J. Phys. E 6, 1077 (1973).

${ }^{7}$ J. P. Macau, J. Jamar, and S. Gardier, IEEE Trans. Nucl. Sci. NS-23, 2049 (1976).

${ }^{8}$ G. E. Iglesias and J. O. McGarity, Rev. Sci. Instrum. 42, 1728 (1971).

${ }^{9}$ W. E. Potter and K. Mauersberger, Rev. Sci. Instrum. 43, 1327 (1972).

${ }^{10}$ D. H. Crandall, J. A. Ray, and C. Cisneros, Rev. Sci. Instrum. 46, 562 (1975).

${ }^{11}$ S. A. Fields, J. L. Burch, and W. A. Oran, Rev. Sci. Instrum. 48, 1076 (1977).
12 J. Fricke, A. Müller, and E. Salzborn, Nucl. Instrum. Methods 175, 379 (1980).

${ }^{13}$ J. P. Ravo, Nucl. Instrum. Methods 211, 7 (1983).

${ }^{14}$ J. Dimeff, A. J. Lieber, and C. N. Burrows, Rev. Sci. Instrum. 37, 1562 (1966).

${ }^{15}$ C. N. Burrows, A. J. Lieber, and V. T. Zaviansteff, Rev. Sci. Instrum. 38, 1477 (1967).

${ }^{16}$ L. D. Gardner, J. L. Kohl, G. P. Lafyatis, A. R. Young, and A. Chutjian, Rev. Sci. Instrum. 57, 2254 (1986).

${ }^{17} \mathrm{~J}$. A. Ray and C. F. Barnett, IEEE Trans. Nucl. Sci. NS-17, 44 (1970).

${ }^{18}$ J. N. Fox, R. L. Fitzwilson, and E. W. Thomas, J. Phys. E 3, 36 (1970).

${ }^{19} \mathrm{~K}$. C. Schmidt, Bendix Electro-Optics Division Technical Application Note 9803 (25 April 1969).

${ }^{20} \mathrm{~J}$. F. O'Hanlon, A User's Guide to Vacuum Technology (Wiley, New York, 1989), pp. 88-93.

${ }^{21}$ Varian Ionization Gauge Instruction Sheet 87-400 095A 377, March 1977.

${ }^{22}$ D. H. Crandall, M. L. Mallory, and D. C. Kocher, Phys. Rev. A 15, 61 (1977).

${ }^{23}$ D. H. Crandall, R. A. Phaneuf, and F. W. Meyer, Phys. Rev. A 19, 504 (1979).

${ }^{24}$ S. Bliman, J. Aubert, R. Geller, B. Jacquot, and D. Van Houtte, Phys. Rev. A 23, 1703 (1981).

${ }^{25}$ D. Ćirić, A. Brazuk, D. Dijkkamps, F. J. de Heers, and H. Winter, J. Phys. B 18, 3639 (1985).

${ }^{26}$ D. W. Savin, Ph.D. thesis, Harvard University, 1994. 